\title{
Análise multivariada de atributos nutricionais associados ao "bitter pit" em maçãs 'Gala'
}

\author{
Cassandro Vidal Talamini do Amarante(1), Daniela Vieira Chaves ${ }^{(1)}$ e Paulo Roberto Ernani(1)
}

(1)Universidade do Estado de Santa Catarina, Centro de Ciências Agroveterinárias, Caixa Postal 281, CEP 88502-970 Lages, SC. E-mail:
amarante@cav.udesc.br, a6dvc@cav.udesc.br, a2pre@cav.udesc.br

Resumo - O objetivo deste trabalho foi identificar atributos nutricionais, quantificados na polpa e na casca dos frutos, que melhor discriminam quanto à severidade de "bitter pit” em maçãs ‘Gala'. Depois de quatro meses de armazenamento em atmosfera normal $\left(0-1{ }^{\circ} \mathrm{C} ; 90-95 \%\right.$ UR), os frutos foram separados em quatro lotes, de acordo com a severidade de incidência de "bitter pit": nula (nenhuma lesão por fruto), baixa (1-2 lesões por fruto), moderada (3-4 lesões por fruto) e alta (5-13 lesões por fruto). Foram determinados os teores de Ca, Mg, K e N na casca e na polpa em frutos individuais (doze frutos de cada lote). A análise univariada mostrou que os frutos com sintomas de "bitter pit" apresentaram menor teor de Ca na casca e na polpa, maior teor de K na casca e maiores valores das relações $\mathrm{K} / \mathrm{Ca}, \mathrm{Mg} / \mathrm{Ca}, \mathrm{N} / \mathrm{Ca},(\mathrm{K}+\mathrm{Mg}) / \mathrm{Ca}$ e $(\mathrm{K}+\mathrm{Mg}+\mathrm{N}) / \mathrm{Ca}$, tanto na casca como na polpa. Todavia, quando todos os atributos nutricionais avaliados na casca e na polpa foram submetidos à análise canônica discriminante (análise multivariada), a melhor discriminação entre frutos sem "bitter pit” e frutos com severidade de incidência de "bitter pit" de baixa a alta é obtida com a relação K/Ca na casca, seguido, em menor grau, pelas relações $\mathrm{Mg} / \mathrm{Ca}$ e N/Ca na polpa.

Termos para indexação: Malus domestica, nutrição mineral, análise canônica discriminante, distúrbio fisiológico, qualidade pós-colheita.

\section{Multivariate analysis of nutritional attributes associated with bitter pit in 'Gala' apples}

\begin{abstract}
The objective of this work was to identify nutritional attributes, assessed in the flesh and skin tissues, that provide a better discrimination regarding bitter pit severity in 'Gala' apples. After four months in regular cold storage $\left(0-1{ }^{\circ} \mathrm{C} ; 90-95 \% \mathrm{RH}\right)$, fruits were segregated into four lots with different levels of bitter pit severity: null (none pit per fruit), low (1-2 pits per fruit), moderate (3-4 pits per fruit), and high (5-13 pits per fruit). Nutritional analysis (Ca, Mg, K and $\mathrm{N}$ ) in the skin and in the flesh tissues were performed on twelve individual fruits of each severity level. The univariate analysis showed that fruits with bitter pit symptoms had lower concentration of $\mathrm{Ca}$ in the skin and flesh tissues, a higher concentration of $\mathrm{K}$ in the skin tissue, and higher values for $\mathrm{K} / \mathrm{Ca}, \mathrm{Mg} / \mathrm{Ca}, \mathrm{N} / \mathrm{Ca},(\mathrm{K}+\mathrm{Mg}) / \mathrm{Ca}$, and $(\mathrm{K}+\mathrm{Mg}+\mathrm{N}) / \mathrm{Ca}$ ratios in the skin and flesh tissues than fruits without bitter pit. However, the canonical discriminant analysis (multivaried analysis) showed that, out of all nutritional attributes assessed in the skin and flesh tissues, K/Ca ratio in the skin provides the best discrimination between fruit with bitter pit and fruit without the disorder, followed by $\mathrm{Mg} / \mathrm{Ca}$ and $\mathrm{N} / \mathrm{Ca}$ ratio in the flesh tissue.

Index terms: Malus domestica, mineral nutrition, canonical discriminant analysis, physiological disorder, postharvest quality.
\end{abstract}

\section{Introdução}

O “bitter pit” é um distúrbio fisiológico verificado em todas as áreas de produção de maçãs do mundo. No Brasil, esse distúrbio ocorre principalmente em maçãs 'Gala', 'Fuji', 'Catarina' e 'Golden Delicious' (Argenta \& Suzuki, 1994; Nachtigall \& Freire, 1998; Amarante et al., 2006). O sintoma primário é uma discreta mancha na polpa, de coloração escura, que se torna desidratada com o tempo, criando assim pequenas depressões na epiderme do fruto (Ferguson \& Watkins, 1989). Apesar de aparecer normalmente durante a fase de frigoconservação, as causas e fatores predisponentes ao "bitter pit" estão presentes no pomar.

Em casos extremos, os sintomas podem aparecer antes mesmo da colheita (Faust \& Shear, 1968; Ferguson \& Watkins, 1989). Cultivares com baixa suscetibilidade, sob condições de crescimento normais, podem apresentar 
alta incidência de "bitter pit" sob circunstâncias agravantes, tais como pequena carga de fruto, verões secos, podas muito severas, adubação nitrogenada excessiva ou colheita antecipada (Ferguson \& Watkins, 1989). Pode haver incidência em até 30\% dos frutos provenientes de pomares mais propícios ao distúrbio, depois de alguns meses de frigoconservação (Basso, 2002).

O baixo conteúdo de Ca nos frutos é o principal fator predisponente à ocorrência de "bitter pit" (Ferguson \& Watkins, 1989; Meheriuk et al., 1994; Amarante et al., 2005). O adequado suprimento de Ca em maçãs retarda a maturação (Poovaiah et al., 1988; Siddiqui \& Bangerth, 1995) e reduz a incidência de doenças (Sharples \& Johnson, 1977) e de diversos distúrbios fisiológicos póscolheita, especialmente o "bitter pit" (Meheriuk et al., 1994). Os efeitos positivos do Ca na preservação da qualidade pós-colheita resultam da sua associação com substâncias pécticas da lamela média e com as membranas celulares, conferindo rigidez aos tecidos e preservando as características de permeabilidade seletiva do sistema de membranas celulares (Poovaiah et al., 1988; Ferguson \& Watkins, 1989).

Além da deficiência de $\mathrm{Ca}$, o desenvolvimento de "bitter pit" está relacionado com elevados conteúdos de outros nutrientes nos frutos, principalmente $\mathrm{Mg}, \mathrm{K} \mathrm{e} \mathrm{N}$ (Ferguson \& Watkins, 1989; Argenta \& Suzuki, 1994; Nachtigall \& Freire, 1998; Amarante et al., 2006). Estes nutrientes apresentam elevada mobilidade nas plantas, ao contrário do $\mathrm{Ca}$, e, portanto, continuam sendo transportados via floema, das folhas para os frutos, depois da fase de divisão celular, favorecendo a ocorrência de "bitter pit” (Tromp, 1975). Relações $\mathrm{K} / \mathrm{Ca},(\mathrm{K}+\mathrm{Mg}) / \mathrm{Ca}$ e N/Ca altas resultam em frutos com maior susceptibilidade ao "bitter pit" (Faust \& Shear, 1968; Ferguson \& Watkins, 1989; Argenta \& Suzuki, 1994; Nachtigall \& Freire, 1998; Basso, 2002; Amarante et al., 2006).

Além das relações nutricionais entre o $\mathrm{Ca}$ e os elementos Mg, K e N, o método de amostragem de tecidos dos frutos pode influenciar o resultado da análise de risco de ocorrência de "bitter pit". O método de amostragem consiste na retirada de uma fatia longitudinal (menos pedúnculo e semente), contendo tecidos de casca e polpa. Todavia, o Ca apresenta grande variação de teor dentro do próprio fruto. $\mathrm{O}$ teor de $\mathrm{Ca}$, em uma seção radial, é superior na casca e centro do fruto, e inferior no córtex: os menores teores estão no córtex exterior do fruto, onde ocorre o "bitter pit" (Terblanche et al., 1979). Existe ainda um gradiente longitudinal, em que o teor de Ca diminui do pedúnculo para o cálice (Lewis \& Martin, 1973; Ferguson \& Watkins, 1983). Portanto, o método tradicional de amostragem (fatia longitudinal de casca+polpa), envolvendo tecidos com teores de nutrientes bastante diferenciados pode reduzir a capacidade de predição do risco de "bitter pit". A amostragem individualizada dos tecidos da casca e da polpa, assim como sua análise nutricional, pode permitir a identificação do tecido e do atributo nutricional que melhor discrimina os frutos quanto à severidade de "bitter pit".

Métodos de análise univariada são empregados visando a identificar diferenças entre grupos de frutos com diferentes graus de severidade de "bitter pit", considerando individualmente cada atributo nutricional (Ferguson \& Watkins, 1989). Estes métodos de análise não consideram o efeito conjunto de todos os atributos nutricionais. A análise estatística multivariada, como é o caso da análise canônica discriminante, é um instrumento que permite a identificação de diferenças entre grupos de frutos com ocorrência variável de "bitter pit", considerando todos os atributos nutricionais (Cruz-Castillo et al., 1994). Por tais características, a análise multivariada representa importante instrumento no estudo e identificação dos atributos nutricionais que melhor discriminam quanto à severidade de "bitter pit", por inexistência de similaridade. Isto pode permitir a eliminação do tempo gasto com a avaliação de atributos nutricionais que expressam baixa eficiência na discriminação de frutos quanto ao grau de ocorrência de "bitter pit".

O objetivo deste trabalho foi identificar nutrientes e relações nutricionais que afetam a severidade de incidência de "bitter pit", em frutos da cultivar Gala, bem como determinar a porção do fruto (polpa ou casca) que relaciona melhor o estado nutricional com a severidade do distúrbio, por meio da utilização comparativa de métodos de análise estatística univariada e multivariada (análise canônica discriminante).

\section{Material e Métodos}

A colheita de frutos foi efetuada em macieiras da cultivar Gala (sobre porta-enxerto Marubakaido), com 13 anos de idade, cultivadas num espaçamento de 4,0x6,5 m, em pomar localizado no Município de Lages, SC, na safra 2000/2001, durante a qual, as plantas receberam oito pulverizações com cloreto de cálcio $(0,5 \%, \mathrm{p} / \mathrm{v})$. 
Depois do armazenamento por quatro meses em atmosfera normal (temperatura de $0-1{ }^{\circ} \mathrm{C}$ e $90-95 \%$ de umidade relativa), os frutos foram divididos em quatro lotes, de acordo com o grau da severidade de "bitter pit”: nulo, nenhuma lesão por fruto; baixo, 1-2 lesões por fruto; moderado, 3-4 lesões por fruto; e alto, 5-13 lesões por fruto. Cada lote foi constituído por doze frutos, nos quais determinaram-se (em $\mathrm{mg} \mathrm{kg}^{-1}$ ), individualmente, os teores de $\mathrm{Ca}, \mathrm{K}, \mathrm{Mg}$ e $\mathrm{N}$, na casca e na polpa. O delineamento experimental foi o inteiramente casualizado, com 12 repetições, cada qual correspondendo a um fruto.

Foi efetuada a remoção da casca de toda a superfície do fruto e utilizado todo o tecido da polpa - região do córtex, exceto o tecido da região carpelar central - de cada fruto. Os tecidos da casca e da polpa dos frutos foram digeridos a $350^{\circ} \mathrm{C}$, usando-se uma mistura de ácido sulfúrico concentrado e água oxigenada 30 volumes. O N foi determinado pelo método semimicro Kjeldahl, como descrito por Tedesco et al. (1995), e os demais nutrientes ( $\mathrm{K}$, Ca e $\mathrm{Mg}$ ) foram determinados por espectrofotometria de emissão induzida por plasma.

Os dados foram submetidos à análise estatística, utilizando o programa SAS, versão 6.12 (SAS Institute, 1990). Foi realizada análise de variância e teste de comparação de médias (Tukey, a 5\% de probabilidade) em relação aos atributos nutricionais quantificados na casca e na polpa, em frutos com diferentes suscetibilidade ao "bitter pit". Os dados foram também submetidos à análise canônica discriminante (ACD), visando a identificar o tecido do fruto (casca ou polpa), bem como os atributos nutricionais que permitem discriminar diferenças quanto ao grau de severidade do “bitter pit” em maçãs ‘Gala'.

O parâmetro adotado na avaliação do efeito de separação, gerada pelos atributos nutricionais, entre os graus de severidade estudados, foi o coeficiente da taxa de discriminação paralela (TDP). O coeficiente da TDP é obtido por meio do produto entre valores dos coeficientes canônicos padronizados (CCP) e os valores dos coeficientes de correlação canônica (r). No coeficiente da TDP, valores de atributos nutricionais positivos indicam efeito de separação entre os graus de severidade de "bitter pit", e os atributos nutricionais com os maiores valores apresentam maior peso na separação entre graus de severidade. Valores negativos expressam efeito de supressão do atributo nutricional na separação entre os graus de severidade de "bitter pit", ou seja, expressam semelhanças entre os índices de severidade. Os valores médios de CCP de frutos correspondentes às diferentes intensidades de susceptibilidade ao "bitter pit” foram comparados por meio do teste de Tukey a $5 \%$ de probabilidade, conforme descrito por CruzCastillo et al. (1994).

\section{Resultados e Discussão}

No tecido da casca, os teores de $\mathrm{Mg}$ e $\mathrm{N}$ não diferiram entre frutos com graus de severidade nula e alta de "bitter pit" (Tabela 1). Frutos com "bitter pit", independentemente da severidade, apresentaram menor teor de Ca e maior teor de K na casca, em relação aos

Tabela 1. Composição química ( $\mathrm{mg} \mathrm{kg}^{-1}$ ) e relações nutricionais na casca e na polpa dos frutos da cultivar Gala com diferentes severidades de incidência de "bitter pit”(1).

\begin{tabular}{|c|c|c|c|c|c|c|c|c|c|}
\hline Severidade $^{(2)}$ & $\mathrm{Ca}$ & $\mathrm{Mg}$ & $\mathrm{K}$ & $\mathrm{N}$ & $\mathrm{K} / \mathrm{Ca}$ & $\mathrm{Mg} / \mathrm{Ca}$ & $\mathrm{N} / \mathrm{Ca}$ & $(\mathrm{K}+\mathrm{Mg}) / \mathrm{Ca}$ & $(\mathrm{K}+\mathrm{Mg}+\mathrm{N}) / \mathrm{Ca}$ \\
\hline & & & & & Casca & & & & \\
\hline Nula & $152 \mathrm{a}$ & $209 a$ & $1.055 \mathrm{~b}$ & $433 a$ & $7,5 \mathrm{~b}$ & $1,5 b$ & $3,0 \mathrm{~b}$ & $9,0 \mathrm{~b}$ & $12,0 \mathrm{~b}$ \\
\hline Baixa & $100 \mathrm{~b}$ & $191 b$ & $1.184 \mathrm{a}$ & $399 a$ & $12,4 \mathrm{a}$ & $2,0 \mathrm{a}$ & $4,1 \mathrm{a}$ & $14,3 a$ & $18,4 \mathrm{a}$ \\
\hline Moderada & $97 b$ & $191 b$ & $1.240 \mathrm{a}$ & $397 \mathrm{a}$ & $13,1 \mathrm{a}$ & $2,0 \mathrm{a}$ & $4,2 \mathrm{a}$ & $15,1 \mathrm{a}$ & $19,3 \mathrm{a}$ \\
\hline Alta & $101 \mathrm{~b}$ & $204 a b$ & $1.339 \mathrm{a}$ & $441 \mathrm{a}$ & $13,7 \mathrm{a}$ & $2,1 \mathrm{a}$ & $4,5 \mathrm{a}$ & $15,8 \mathrm{a}$ & $20,2 \mathrm{a}$ \\
\hline CV (\%) & 33,8 & 12,6 & 19,6 & 18,6 & 41,1 & 27,0 & 18,6 & 38,5 & 35,7 \\
\hline & & & & & Polpa & & & & \\
\hline Nula & $38,8 \mathrm{a}$ & $45,1 \mathrm{a}$ & $1.127 \mathrm{a}$ & $230 \mathrm{a}$ & $31,3 b$ & $1,2 b$ & $6,3 b$ & $32,5 b$ & $38,8 b$ \\
\hline Baixa & $29,5 b$ & $42,5 \mathrm{a}$ & $1.168 \mathrm{a}$ & $238 \mathrm{a}$ & $41,3 \mathrm{a}$ & $1,5 \mathrm{a}$ & $8,4 \mathrm{a}$ & $42,8 \mathrm{a}$ & $51,2 \mathrm{a}$ \\
\hline Moderada & $28,8 \mathrm{~b}$ & $40,0 \mathrm{~b}$ & $1.182 \mathrm{a}$ & $244 a$ & $41,8 \mathrm{a}$ & $1,4 \mathrm{a}$ & $8,7 \mathrm{a}$ & $43,2 \mathrm{a}$ & $51,9 \mathrm{a}$ \\
\hline Alta & $29,6 \mathrm{~b}$ & $42,8 \mathrm{a}$ & $1.104 \mathrm{a}$ & $262 \mathrm{a}$ & $38,3 \mathrm{a}$ & $1,5 \mathrm{a}$ & $8,8 \mathrm{a}$ & $39,8 \mathrm{a}$ & $48,6 \mathrm{a}$ \\
\hline $\mathrm{CV}(\%)$ & 29,6 & 17,8 & 16,3 & 24,1 & 33,0 & 20,1 & 33,8 & 32,3 & 31,0 \\
\hline
\end{tabular}

(1)Médias nas colunas, em cada tecido do fruto (casca ou polpa), seguidas pela mesma letra, não diferem entre si pelo teste de Tukey a 5\% de probabilidade. ${ }^{(2)}$ Severidade: nula: nenhuma lesão por fruto; baixa: 1-2 lesões por fruto; moderada: 3-4 lesões por fruto; e alta: 5-13 lesões por fruto. 
frutos sem "bitter pit" (Tabela 1). Todas as relações nutricionais estudadas no tecido da casca [K/Ca, Mg/Ca, N/Ca, $(\mathrm{K}+\mathrm{Mg}) / \mathrm{Ca}$ e $(\mathrm{K}+\mathrm{Mg}+\mathrm{N}) / \mathrm{Ca}]$ revelaram menores valores para frutos sem "bitter pit" do que para frutos com severidade baixa a alta de "bitter pit” (Tabela 1).

Quanto ao tecido da polpa, somente os teores de Ca foram maiores nos frutos sem "bitter pit” em relação a frutos com severidade baixa a alta (Tabela 1). O teor de K, Mg e N não variou entre frutos com diferentes severidades de "bitter pit". No entanto, frutos com severidade baixa, moderada e alta, apresentaram maiores valores das relações $\mathrm{K} / \mathrm{Ca}, \mathrm{Mg} / \mathrm{Ca}$, N/Ca, $(\mathrm{K}+\mathrm{Mg}) / \mathrm{Ca}$ e $(\mathrm{K}+\mathrm{Mg}+\mathrm{N}) / \mathrm{Ca}$, em relação a frutos sem "bitter pit" (Tabela 1). Frutos com valores das relações $(\mathrm{K}+\mathrm{Mg}) /$ $\mathrm{Ca}=32,5, \mathrm{~K} / \mathrm{Ca}=31,3, \mathrm{Mg} / \mathrm{Ca}=1,2, \mathrm{~N} / \mathrm{Ca}=6,3 \mathrm{e}$ $(\mathrm{K}+\mathrm{Mg}+\mathrm{N}) / \mathrm{Ca}=38,8$, na polpa, mostraram-se isentos de "bitter pit".

Em maçãs 'Golden Delicious', frutos com relação $(\mathrm{K}+\mathrm{Mg}) / \mathrm{Ca}$, na polpa fresca, na colheita, superior a 32 apresentaram maior severidade de "bitter pit" (Nachtigall \& Freire, 1998). No presente trabalho, frutos com relação $(\mathrm{K}+\mathrm{Mg}) / \mathrm{Ca}$, no tecido da polpa, igual ou superior a 32,8 apresentaram “bitter pit” (Tabela 1). Argenta \& Suzuki (1994) verificaram menor incidência de "bitter pit" em frutos com relação $(\mathrm{K}+\mathrm{Mg}) / \mathrm{Ca}$ menor do que 27 no tecido de casca+polpa de maçãs cultivar Gala. Considerando que os autores amostraram casca+polpa, e que a casca apresenta menor valor da relação $(\mathrm{K}+\mathrm{Mg}) / \mathrm{Ca}$ em relação à polpa (Tabela 1$)$, é esperado um valor menor dessa relação, quando realizada a amostragem de casca+polpa em comparação à amostragem apenas de polpa.

Na ACD, a primeira função discriminante canônica explicou 84,3\% da variação total, enquanto a segunda e a terceira funções explicaram apenas 11,2 e $4,5 \%$ da variação total, respectivamente. Dessa forma, como a primeira função discriminante canônica (função canônica discriminante $1 ; \mathrm{FCD}_{1}$ ) explicou a maior parte da variação total, apenas esta foi considerada na análise multivariada dos dados.

O teste estatístico multivariado Wilks Lambda mostrou diferenças significativas entre frutos com diferentes graus de severidade de "bitter pit" $(\mathrm{p}<0,0001)$ para a $\mathrm{FCD}_{1}$. A FCD $\mathrm{F}_{1}$ apresentou correlação canônica de 0,7226 , indicando elevada associação entre os atributos nutricionais e a severidade de "bitter pit".
A relação K/Ca na casca apresentou o maior valor de coeficiente da TDP (Tabela 2), indicando que, entre todos os atributos nutricionais avaliados, nos tecidos da casca e da polpa, este é o que melhor discrimina entre frutos sem "bitter pit" e frutos com severidade baixa a alta. Em menor grau de importância, quanto a essa discriminação, estão as relações $\mathrm{Mg} / \mathrm{Ca}$ e N/Ca na polpa (Tabela 2). O teor isolado de Ca, na casca e na polpa, quando considerado em uma análise multivariada, não se mostrou relevante na discriminação entre lotes de frutos com e sem "bitter pit”. Isto confirma observações realizadas por outros autores, mostrando que relações entre teores de Ca e dos nutrientes Mg e K são melhores indicadores de suscetibilidade ao "bitter pit" do que apenas os teores de Ca (Faust \& Shear, 1968; Ferguson \& Watkins, 1989; Argenta \& Suzuki, 1994; Nachtigall \& Freire, 1998; Amarante et al., 2006).

A ACD demonstra que o teor isolado de $\mathrm{Ca}$ (na casca ou na polpa), comparativamente aos valores de suas relações com os elementos $\mathrm{K}$ e Mg, apresenta maior variabilidade entre frutos de um mesmo lote de severidade e/ou menor variabilidade entre frutos de diferentes lotes de severidade de "bitter pit". Portanto, apesar de a análise univariada apontar teores de $\mathrm{Ca}$, na casca e polpa, significativamente maiores em frutos sem "bitter pit" do que em frutos com sintomas do distúrbio (Tabela 1), a análise multivariada demonstra pouca ou nenhuma capacidade desse nutriente em discriminar lotes dos frutos, sendo a relação K/Ca, na casca, melhor indicada (Tabela 2).

A representação gráfica entre os CCP das funções canônicas discriminantes 1 e 2 mostra uma separação entre frutos sem "bitter pit" e frutos com severidade baixa a alta do distúrbio (Figura 1). Houve diferença altamente significativa $(p<0,0001)$ entre os valores médios

Tabela 2. Coeficiente da taxa de discriminação paralela para a função canônica discriminante 1 , referente às análises dos nutrientes e suas relações na casca e na polpa em maçãs 'Gala'.

\begin{tabular}{lrr}
\hline Atributo & Casca & \multicolumn{1}{c}{ Polpa } \\
\hline $\mathrm{Ca}$ & $-0,0722$ & $-0,1631$ \\
$\mathrm{Mg}$ & $-0,0940$ & 0,1757 \\
$\mathrm{~K}$ & $-0,4216$ & 0,0320 \\
$\mathrm{~N}$ & 0,0053 & $-0,0445$ \\
$\mathrm{~K} / \mathrm{Ca}$ & 2,2786 & $-0,5388$ \\
$\mathrm{Mg} / \mathrm{Ca}$ & $-0,8273$ & 0,4845 \\
$\mathrm{~N} / \mathrm{Ca}$ & $-0,1293$ & 0,3148 \\
$(\mathrm{~K}+\mathrm{Mg}) / \mathrm{Ca}$ & 0,0000 & 0,0000 \\
$(\mathrm{~K}+\mathrm{Mg}+\mathrm{N}) / \mathrm{Ca}$ & 0,0000 & 0,0000 \\
\hline
\end{tabular}


de CCP da FCD 1 entre frutos sem "bitter pit" e frutos com severidade baixa a alta (Tabela 3). Frutos com severidades baixa, moderada e alta apresentaram valores médios maiores de CCP em relação a frutos sem "bitter pit”, principalmente como resultado dos valores maiores da relação K/Ca na casca.

Os resultados mostram que, em maçãs 'Gala', a análise nutricional, na casca e na polpa, discrimina apenas frutos com e sem sintomas de "bitter pit”, não permitindo a separação quanto à severidade do distúrbio em frutos com sintoma (Tabelas 1 e 3; Figura 1). Isto reflete a dificuldade de quantificação da severidade de "bitter pit" com base na análise nutricional dos frutos. Neste trabalho,

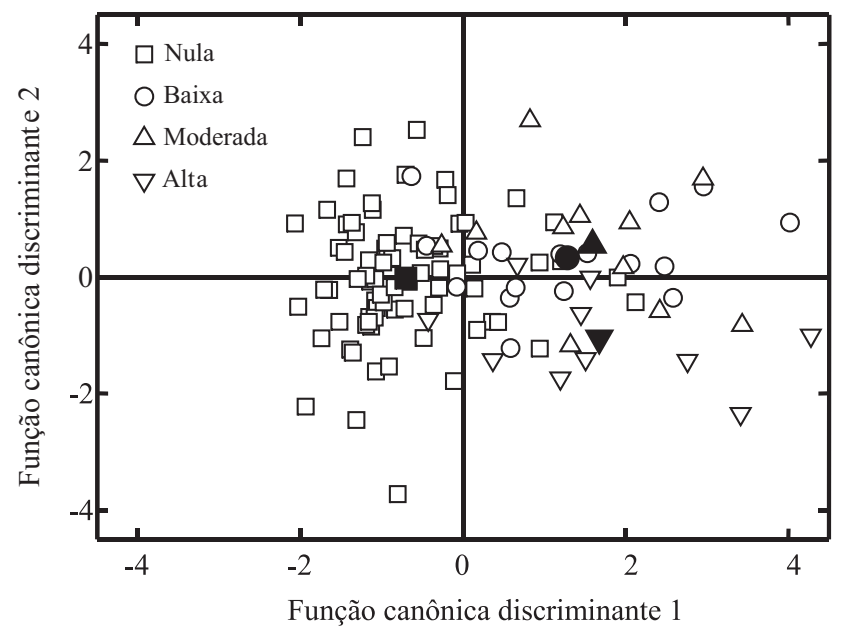

Figura 1. Coeficientes canônicos padronizados das funções canônicas discriminantes 1 e 2, em maçãs ‘Gala' com diferentes graus de severidade de "bitter pit", considerando todos os atributos nutricionais avaliados na polpa e na casca. Símbolo cheio representa o valor médio de CCP para cada grau de severidade de "bitter pit".

Tabela 3. Valores médios dos coeficientes canônicos padronizados (CCP) para a função canônica discriminante 1 , referente às análises nutricionais de maçãs 'Gala' com diferentes graus de severidade de "bitter pit”.

\begin{tabular}{lr}
\hline Severidade $^{(1)}$ & $\mathrm{CCP}^{(2)}$ \\
\hline Nula & $-0,7089 \mathrm{~b}$ \\
Baixa & $1,2778 \mathrm{a}$ \\
Moderada & $1,5925 \mathrm{a}$ \\
Alta & $1,6765 \mathrm{a}$ \\
\hline
\end{tabular}

(1)Severidade nula: nenhuma lesão por fruto; baixa: 1-2 lesões por fruto; moderada: 3-4 lesões por fruto; e alta: 5-13 lesões por fruto. (2)Médias, na coluna, seguidas da mesma letra não diferem entre si pelo teste de Tukey a $5 \%$ de probabilidade. foram amostrados, em grande parte, tecidos com conteúdo nutricional não predisponente à ocorrência de "bitter pit”. Manchas de "bitter pit” ocorrem em grupos específicos de células, que apresentam desequilíbrio nos teores de $\mathrm{Ca}$, relativamente aos de $\mathrm{Mg}, \mathrm{K}$ e $\mathrm{N}$ (Burmeister \& Dilley, 1994). Isto resulta em baixa capacidade de segregação entre frutos com severidade baixa a alta, com base na análise nutricional, sendo possível apenas uma separação em relação a frutos não suscetíveis. Para produtores de maçãs, interessa mais a presença ou ausência de "bitter pit”, não sendo relevante a severidade. As normas e padrões do Ministério da Agricultura prevêem valores máximos de tolerância de frutos com "bitter pit" de 5\% para o tipo extra, 10\% para o tipo especial e $20 \%$ para o tipo comercial (Basso, 2002).

Além da variabilidade intrínseca entre frutos amostrados, para a análise nutricional no pomar estádio de maturação do fruto, carga de frutos, relação frutos/área foliar, posição do fruto na planta, vigor da planta (Ferguson \& Watkins, 1989) -, existe variabilidade nas quantidades relativas de $\mathrm{Ca}, \mathrm{Mg}, \mathrm{K}$ e $\mathrm{N}$ em diferentes tecidos de frutos individuais (Faust \& Shear, 1968). No entanto, a ACD mostra que é possível obter boa discriminação entre maçãs 'Gala' com e sem "bitter pit” por meio da utilização da casca, mas não da polpa dos frutos, na análise nutricional. Como o desenvolvimento das lesões de "bitter pit" inicia-se no tecido logo abaixo da epiderme e estendese até algumas camadas de células em direção à polpa, a análise nutricional da casca do fruto passa a ter maior valor na predição de ocorrência de "bitter pit" do que a análise do tecido da polpa (Amarante et al., 2005, 2006). Sendo assim, em maçãs 'Gala', a casca é a porção do fruto mais indicada para a amostragem e a quantificação dos teores de $\mathrm{Ca}$ e $\mathrm{K}$, visando à segregação de frutos suscetíveis ao "bitter pit". Frutos com relação K/Ca no tecido da casca igual ou superior a 12,4 são suscetíveis ao "bitter pit”.

\section{Conclusões}

1. A ocorrência de "bitter pit" na cultivar Gala é conseqüência de baixos teores de Ca na casca e na polpa e de altos teores de $\mathrm{K}$ na casca.

2. A amostragem da casca é mais indicada para a análise nutricional visando a caracterizar a ocorrência de "bitter pit” em maçãs ‘Gala'. 
3. Maçãs 'Gala' com relação K/Ca no tecido da casca igual ou superior a 12,4 são suscetíveis ao "bitter pit”.

\section{Agradecimentos}

Ao CNPq e à Capes, pelo apoio financeiro.

\section{Referências}

AMARANTE, C.V.T. do; CHAVES, D.V.; ERNANI, P.R. Composição mineral e severidade de bitter pit em maçãs 'Catarina'. Revista Brasileira de Fruticultura, v.28, p.51-54, 2006.

AMARANTE, C.V.T. do; ERNANI, P.R.; CHAVES, D.V. Fruit infiltration with magnesium is a feasible way to predict bitter pit susceptibility in 'Gala' apples grown in Southern Brazil. Acta Horticulturae, v.682, p.1271-1274, 2005.

ARGENTA, L.C.; SUZUKI, A. Relação entre teores minerais e freqüência de bitter pit em maçã cv. Gala no Brasil. Revista Brasileira de Fruticultura, v.16, p.267-277, 1994.

BASSO, C. Distúrbios fisiológicos. In: EPAGRI. A cultura da macieira. Florianópolis, 2002. p.609-636.

BURMEISTER, D.M.; DILLEY, D.R. Correlation of bitter pit on Northern Spy apples with bitter pit-like symptoms induced by $\mathrm{Mg}^{2+}$ salt infiltration. Postharvest Biology and Technology, v.4, p.301308, 1994.

CRUZ-CASTILLO, J.G.; GANESHANANDAM, S.; MACKAY, B.R.; LAWES, G.S.; LAWOKO, C.R.O.; WOOLLEY, D.J. Applications of canonical discriminant analysis in horticultural research. HortScience, v.29, p.1115-1119, 1994.

FAUST, M.; SHEAR, C.B. Corking disorders of apple: a physiological and biochemical review. Botanical Review, v.34, p.441-469, 1968.

FERGUSON, I.B.; WATKINS, C.B. Bitter-pit in apple fruit. Horticultural Reviews, v.11, p.289-355, 1989.

FERGUSON, I.B.; WATKINS, C.B. Cation distribution and balance in apple fruit in relation to calcium treatments for bitter pit. Scientia Horticulturae, v.19, p.301-310, 1983.
LEWIS, T.L.; MARTIN, D. Longitudinal distribution of applied calcium, and of naturally occurring calcium, magnesium, and potassium in Merton apple fruits. Australian Journal of Agricultural Research, v.24, p.363-371, 1973.

MEHERIUK, M.; PRANGE, R.K.; KIDSTER, P.D.; PORRITT, S.W. Postharvest disorders of apples and pears. Ottawa: Agriculture and Agri-Food Canada, 1994. 67p. (Agriculture and AgriFood Canada. Publication, 1737/E).

NACHTIGALL, G.R.; FREIRE, C.J.S. Previsão da incidência de "bitter pit” em maçãs através dos teores de cálcio em folhas e frutos. Revista Brasileira de Fruticultura, v.20, p.158-166, 1998.

POOVAIAH, B.W.; GLENN, G.M.; REDDY, A.S.N. Calcium and fruit softening: physiology and biochemistry. Horticultural Reviews, v.10, p.107-152, 1988.

SAS INSTITUTE (Cary, Estados Unidos). Doing more with SAS/ ASSIST software: version 6. Cary, 1990. 789p.

SHARPLES, R.O.; JOHNSON, D.S. The influence of calcium on senescence changes in apples. Annals of Applied Biology, v.85, p.450-453, 1977.

SIDDIQUI, S.; BANGERTH, F. Effect of pre-harvest application of calcium on flesh firmness and cell-wall composition of apples influence of fruit size. Journal of Horticultural Science, v.70, p.263-269, 1995.

TEDESCO, M.J.; GIANELLO, C.; BISSANI, C.A.; BOHNEN, H.; VOLKWEISS, S.J. Análise do solo, planta e outros materiais. 2.ed. Porto Alegre: UFRGS, Departamento de Solos, 1995. 174p. (Boletim técnico, 5).

TERBLANCHE, J.H.; GÜRGEN, K.H.; PEINAR,W.J. Concentration gradients of $\mathrm{K}, \mathrm{Ca}$ and $\mathrm{Mg}$ in Golden Delicious apples with reference to bitter pit. Deciduous Fruit Grower, v.29, p.7679, 1979.

TROMP, J. The effect of temperature on growth and mineral nutrition of fruits of apple, with special reference to calcium. Physiologia Plantarum, v.33, p.87-93, 1975.

Recebido em 31 de março de 2005 e aprovado em 6 de dezembro de 2005 